\title{
Racial/Ethnic Differences in Burnout: a Systematic Review
}

\author{
Jourdyn A. Lawrence ${ }^{1,2,3}$ (D) Brigette A. Davis ${ }^{2,3} \cdot$ Thea Corbette $^{1} \cdot$ Emorcia V. Hill $^{1} \cdot$ David R. Williams $^{2,4}$. \\ Joan Y. Reede ${ }^{1,2,5}$
}

Received: 26 October 2020 / Revised: 14 December 2020 / Accepted: 16 December 2020 / Published online: 11 January 2021

(C) W. Montague Cobb-NMA Health Institute 2021

\begin{abstract}
Background Awareness of burnout and its implications within the medical field has been growing. However, an understanding of the prevalence and consequences of burnout among underrepresented minority (URM), specifically underrepresented minority in medicine (UiM) populations, is not readily available.

Objective To examine literature investigating burnout among UiM compared to non-UiM, with particular attention to which measures of burnout are currently being used for which racial/ethnic groups.

Methods The authors identified peer-reviewed articles, published in English through systematic examination using PubMed, PsycINFO, Countway Discovery Medicine, and Web of Science databases. Studies meeting the inclusion criteria were summarized and study quality was assessed.

Results Sixteen studies assessing racial/ethnic differences in burnout were eligible for inclusion. Nearly all studies were crosssectional $(n=15)$ in design and conducted among populations in North America $(n=15)$. Most studies examined burnout among medical students or physicians and used the Maslach Burnout Inventory. Differences in burnout among UiM and non-UiM are inconclusive, although several studies have nuanced findings.

Conclusion Increased focus on burnout measurement, conceptualization, and mitigation among UiM populations may be useful in improving recruitment, retention, and thriving.
\end{abstract}

Keywords Burnout $\cdot$ Underrepresented minority $\cdot$ Emotional exhaustion $\cdot$ Measurement

\section{Introduction}

Medical staff and faculty in academic medicine experience an alarmingly high level of stress [1]. Their large and expanding workload requires managing increasing institutional demands, uncertainty in research endeavors, patient care requirements, and expectations regarding student and/or trainee education

Jourdyn A. Lawrence

jlawrence@g.harvard.edu

1 Office for Diversity Inclusion and Community Partnership, Harvard Medical School, Boston, MA, USA

2 Department of Social and Behavioral Sciences, Harvard T.H. Chan School of Public Health, Harvard University, 677 Huntington Ave, Boston, MA 02115, USA

3 François-Xavier Bagnoud Center for Health and Human Rights, Harvard University, Boston, MA, USA

4 African and African American Studies, Harvard University, Cambridge, MA, USA

5 Massachusetts General Hospital, Boston, MA, USA and administrative tasks. These forms of occupational stress, combined with the challenges of balancing work and family responsibilities, have been associated with reports of symptoms of depression, anxiety, work strain, and discontent [2-6]. Such occupational stress may negatively affect medical staff and faculty well-being and increase the prevalence of burnout [7-9].

Burnout has come to be described as emotional exhaustion, loss of work interest, frustration with feeling ineffective, and the tendency to see people as objects rather than humans [10, 11]. Though burnout is common in a multitude of professions, it is well documented and prevalent among medical professionals and has increasingly become a topic of focus $[6,12$, 13]. Among faculty in US medical schools, it is estimated that approximately $29 \%$ of faculty reported experiencing one or more symptoms of burnout [14], though there have been varying estimates of prevalence among medical students, residents, and early-career physicians $[12,13]$. Burnout has negative effects on both work and personal life. Research suggests that burnout is associated with a decrease in professionalism, productivity and quality of medical care, as well as an increase in medical mistakes and intent to leave $[4,11,15,16]$. 
Additionally, there are serious personal consequences to burnout, including relationship issues, alcohol dependence, and suicidal ideation $[5,6,11,17,18]$.

The conditions which increase the likelihood of burnout among medical professionals, such as stress, pressure to succeed, and experiences with interpersonal discrimination, vary across populations, particularly by race/ethnicity. For instance, focus groups from a sample of medical students around the USA found that lack of cultural representation, racial discrimination, and stereotyping are perceived barriers to success among students self-identifying as Black, Latinx, and Asian [19]. Another nationally representative sample of medical school students found that $64 \%$ reported attending an institution with a negative racial climate, and $81 \%$ reported witnessing someone else being discriminated against [20]. These incidents were associated with greater distress and depression scores than individuals who did not report these events. Evidence suggests these experiences continue throughout training and into the professional setting. Nonwhite faculty in academic medicine report discrimination within their professional/academic environments [21, 22], with personal experiences of discrimination associated with lower career satisfaction [21].

Before June 2003, the Association of American Medical Colleges (AAMC) defined underrepresented minority (URM) as Black Americans (i.e., African American, CaribbeanAmerican), Latinx Americans (i.e., Mexican American, Puerto Ricans), and Native Americans (i.e., American Indians, Alaska Natives, and Native Hawaiians) [23]. Since 2003, the AAMC utilizes underrepresented in medicine (UiM) to refer to "racial and ethnic populations that are underrepresented in the medical profession relative to their numbers in the general population" [23]. Given that the focus of this study is UiM, we will utilize UiM throughout and only use URM when used by the authors themselves.

Data from faculty responses to an AAMC survey on faculty engagement indicate that approximately $35 \%$ of URM women and $21 \%$ of URM men faculty reported experiencing symptoms of burnout $[14,24]$. Such prevalence of burnout among UiM may have implications for intent to leave the position and work satisfaction [14] and suggests a need to further understand burnout among UiM to mitigate its effects and identify points for intervention. Though there are efforts to prevent, alleviate, and increase awareness about burnout, there remains little focus on URM, specifically underrepresented in medicine (UiM) [24].

\section{Burnout Conceptualization}

While the present study is not intended as an extensive overview of relevant theories related to burnout, an understanding of how this concept has been theorized is crucial for examining burnout's measurement, pitfalls, and areas for future work, specifically for UiM populations.

In 1975, Freudenberger defined burnout as symptoms resulting from "excessing demands on energy, strength, or resources" [25]. Building on this, Maslach and Jackson developed the Maslach Burnout Inventory (MBI) in 1981, describing burnout as a syndrome of "emotional exhaustion and cynicism" that is accompanied by a "tendency to evaluate oneself negatively, particularly with regard to one's work" [11]. The MBI includes three subscales, including depersonalization/ cynicism (i.e., withdrawal and inappropriate interactions with others), personal accomplishment (i.e., self-efficacy, success), and emotional exhaustion (i.e., emotional and psychological depletion, affective aspects [26]). The original presentation of the MBI, and several articles that followed, found the MBI to exhibit high validity and reliability; they also found the MBI to be useful across several occupational fields [27] and to be associated with job satisfaction, burnout, and assessments of burnout by faculty [28].

Additional tools to operationalize burnout were developed after researchers observed several limitations in the MBI. For example, (1) there is little to no variation in the framing of emotional exhaustion and depersonalization items, such that all items are negatively worded in these subscales, but personal accomplishment items are all positively framed [29]; (2) personal accomplishment may be seen as a precursor or result of burnout, thus emotional exhaustion and depersonalization remain as key constructs of burnout; and (3) some items may not be seen as relevant to populations outside of the USA [30].

Demerouti and colleagues introduced the Oldenburg Burnout Inventory (OLBI) to address the issue regarding the framing of items in the MBI (e.g., only negatively worded items in the emotional exhaustion and depersonalization subscales) [31]. Incorporating positively and negative worded items, the OLBI was created to examine both ends of the burnout continuum. The scale examines burnout in two dimensions, focusing on measures of affective, cognitive, and physical aspects of exhaustion and disengagement from work (e.g., lack of engagement with work) in order to be applicable to a broader range of occupational workers [29]. The OLBI omits the concept of personal accomplishment as a separate component of burnout as presented in the MBI, due to evidence suggesting that emotional exhaustion and depersonalization develop together, while feelings of personal accomplishment (or the lack thereof) were found to develop independently [31]. Additionally, Demerouti and colleagues identified literature that suggested that personal accomplishment could be conceptualized as a consequence of burnout [32], a personality characteristic (e.g., "self-efficacy"). Additional analysis by Halbesleben and Demerouti found the OLBI to be a valid alternative to the MBI, specifically as it relates to examining a more comprehensive assessment of burnout that also encompasses engagement [33]. 
Kristensen and colleagues introduced the Copenhagen Burnout Inventory (CBI) in 2005 [30]. The authors developed the CBI in response to several issues noticed in the MBI, including, but not limited to, (1) an unclear definition of the MBI and burnout (i.e., the definition implies that all three dimensions [emotional exhaustion, depersonalization, and reduced personal accomplishment] constitute burnout; however, the MBI operationalizes each measure independently and the items are not combined to create a single score) and (2) the inaccessibility of the MBI questionnaires due to their commercial distribution [30]. The CBI conceptualizes the core components of burnout as fatigue and exhaustion, measuring "the degree of physical and psychological fatigue and exhaustion" in three domains: personal, work-related, and client-related burnout [30]. Similar to the MBI, the CBI only includes negatively framed items with the exception of one item in the work-related burnout domain. While the domains exhibit high reliability, issues were raised with the examination of burnout as a singular construct and the similarity of fatigue and exhaustion in the CBI to chronic fatigue [34].

Though emphasis is placed on the MBI, OLBI, and CBI given their frequency of use, burnout has also been operationalized using several measures including the Utrecht Burnout Scale [35], the Professional Quality of Life/Compassion Satisfaction/Fatigue Self-Test [36], the Burnout Measure [37], single items, and investigator-developed measures. However, the utility of these measures has not been examined among UiM specifically.

In this context, the present systematic review aims to examine literature investigating burnout among UiM. We focus on what measures are currently being utilized and among which racial/ethnic groups. Through assessment of the literature, we characterize what is known regarding the prevalence and measurement of burnout among UiM and suggest additional points of consideration regarding the unique experiences, expectations, and stressors that may distinguish factors contributing to burnout and occupational stress for UiM. When available, we also report results disaggregated by race/ethnicity.

\section{Methods}

Following the Preferred Reporting Items for Systematic Reviews and Meta-Analyses (PRISMA) guidelines, we systematically reviewed articles examining racial and/or ethnic differences in burnout among medical students, residents/interns, physicians, and university faculty.

\section{Search Strategy}

Articles were located using PubMed, PsycINFO, Countway Discovery Medicine, and Web of Science databases. The full list of search terms used, by database, is included in Table 1 . Our initial search of the above databases, conducted on July 30, 2018, included all of the terms listed in Table 1, with the exception of clinician-educator. This initial search yielded 1774 studies upon the removal of duplicates. An updated search was conducted on August 3, 2018, including the term clinician-educator, yielding 49 additional studies after the removal of duplicate literature.

\section{Study Selection}

Our search strategy yielded 1823 studies that underwent title and abstract screening to determine their relevance to the present research. Two reviewers (J.A.L. and B.A.D.) conducted title and abstract screening independently, using an online systematic review software - Covidence [38]. Articles appearing relevant for full-text review were also independently screened by two reviewers (J.A.L. and B.A.D). Discordance in decisions was resolved by discussion and consensus. Bibliographies of relevant literature were also searched for additional articles.

\section{Inclusion Criteria}

Studies were considered eligible for inclusion if they were empirical quantitative analyses, assessed burnout among the above fields/roles, and were published in English. No date limits were applied. Exclusion criteria included focus on populations other than those listed above, no assessment of burnout, not a quantitative analysis, and no assessments by race and/or ethnicity.

\section{Quality Assessment}

We conducted quality assessment using the Quality Assessment Tool for Observational Cohort and CrossSectional Studies available through the National Heart, Lung, and Blood Institute (NHLBI) [39]. The tool contains 14 "yes" or "no" questions that are used to examine potential sources of bias across several dimensions including sample selection, exposure and outcome assessment, and adjustment for potential sources of confounding that are used to assign a rating of good, fair, or poor. Ratings were independently assigned by two authors (B.A.D. and J.A.L) and assessed the quality of each article deemed eligible for inclusion. 
Table 1 Search terms utilized by database

\begin{tabular}{|c|c|}
\hline Database & Search terms \\
\hline PubMed & 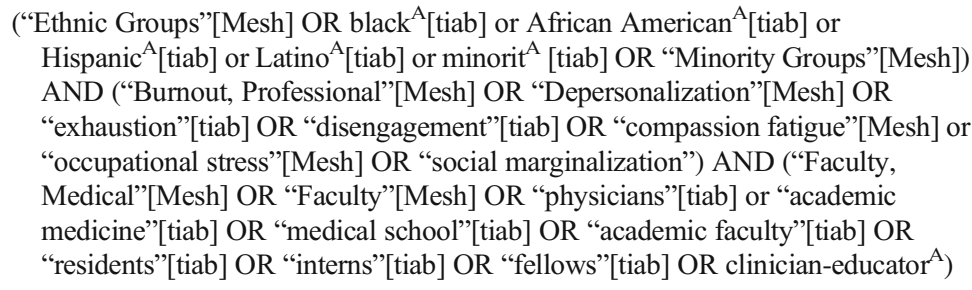 \\
\hline PsycINFO & 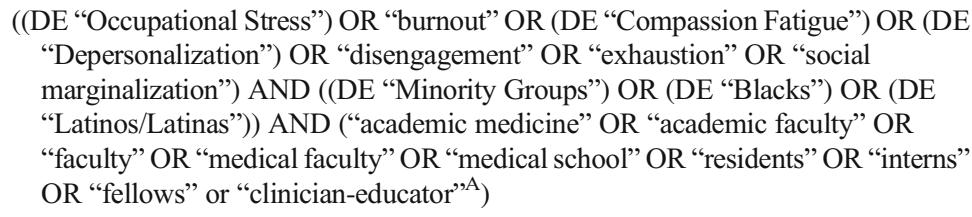 \\
\hline $\begin{array}{l}\text { Countway Discovery } \\
\text { Medicine }\end{array}$ & $\begin{array}{l}\text { (Ethnic Group }{ }^{\mathrm{A}} \text { OR black }{ }^{\mathrm{A}} \text { or African American }{ }^{\mathrm{A}} \text { or Hispanic }{ }^{\mathrm{A}} \text { or Latino }{ }^{\mathrm{A}} \text { or Asian } \\
\text { OR Minority Group }{ }^{\mathrm{A}} \text { ) AND (Burnout OR Depersonalization OR exhaustion OR } \\
\text { disengagement OR "compassion fatigue" or "occupational stress" OR "social } \\
\text { marginalization") AND ("medical faculty" OR Faculty OR "academic medicine" } \\
\text { OR physicians OR "medical school" OR "academic faculty" OR "residents" OR } \\
\text { "interns" OR "fellows" OR "clinician-educator"A) }\end{array}$ \\
\hline Web of Science & $\begin{array}{l}\text { (Ethnic Group }{ }^{\mathrm{A}} \text { OR black } \text { or African American }^{\mathrm{A}} \text { or Hispanic } \text { A }^{\mathrm{A}} \text { or Latino }{ }^{\mathrm{A}} \text { or Asian } \\
\text { OR Minority Group }{ }^{\mathrm{A}} \text { ) AND (Burnout OR Depersonalization OR exhaustion OR } \\
\text { disengagement OR "compassion fatigue" or "occupational stress" OR "social } \\
\text { marginalization") AND ("medical faculty" OR Faculty OR "academic medicine" } \\
\text { OR physicians OR "medical school" OR "academic faculty" OR "residents" OR } \\
\text { "interns" OR "fellows" OR "clinician-educator"A) }\end{array}$ \\
\hline
\end{tabular}

${ }^{\mathrm{A}}$ Term added in the August 3 search

\section{Results}

The initial result of our searches yielded 3540 titles of which 1717 were excluded as duplicates. This left 1823 studies that underwent title and abstract screening. Full-text review was completed among 166 studies. Sixteen studies were considered eligible for inclusion. The study selection process is illustrated in Fig. 1.

Studies deemed eligible for inclusion examined differences in burnout among racial/ethnic groups in varying positions (e.g., physicians, residents, students). Table 2 provides the descriptive statistics of the studies, with a brief summary of associations observed presented in Table 3. All studies which met the inclusion criteria were published between 2004 and 2016. Nearly all studies were cross-sectional $(n=15)$ in design and conducted among populations in North America $(n=15)$. Most studies examined burnout among medical students or physicians and used the Maslach Burnout Inventory, though other instruments used included the Copenhagen Burnout Inventory, the Professional Quality of Life/Compassion Satisfaction/ Fatigue Self-Test, or single-item measures. Nine of the 12 studies that reported sufficient data to ascertain racial/ethnic demographics had populations comprising $\geq 50 \%$ of white or nonMinority samples. Four studies did not provide counts or percentages of the racial/ethnic composition of the samples.
Findings from eligible studies are grouped based upon the population examined.

\section{Faculty}

Of the 16 studies, only one study focused specifically on racial/ethnic differences in burnout among faculty in academic medicine. The cross-sectional analysis, conducted by Pololi and colleagues among a sample of 2218 faculty (512 UiM) identified from the AAMC list of academic health centers, found no statistically significant difference in faculty vitality (i.e., perceptions of being energized by work, looking forward to work, feeling satisfied by work, being proud to work at the institution or feeling burnt out [reverse coded]) by UiM status [40]. Additionally, there was no statistically significant difference in vitality by rank (i.e., professor, associate professor, or assistant professor) or role (i.e., clinician, researcher, administrator, or educator).

Two studies examined racial/ethnic differences in burnout among university faculty. Primack et al.'s analysis of racial differences in burnout found early-career, URM clinical investigators to have higher prevalence of burnout (30\%) compared to white $(18 \%)$ and Asian (3\%) investigators $(p=0.02)$ [41]. In a study among full-time university faculty at one university on the west coast, Lackritz found no statistically 
Fig. 1 Study identification process

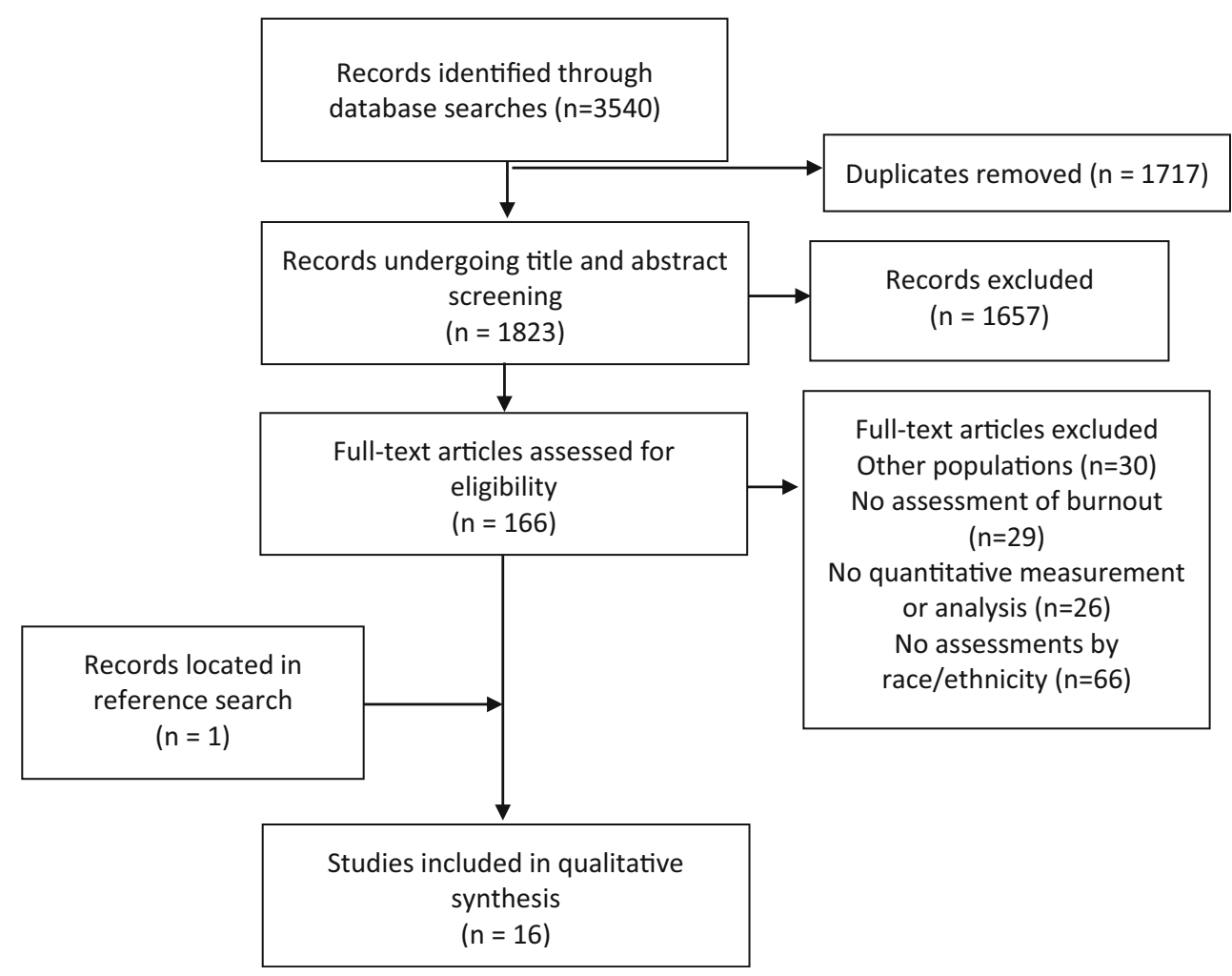

Table 2 Characteristics of the 16 papers included in this systematic review

\begin{tabular}{ll}
\hline & $N,(\%)$ \\
\hline Populations & $3(18.8)$ \\
Faculty & $5(31.3)$ \\
Physicians & $1(6.3)$ \\
Residents/trainees & $6(37.5)$ \\
Medical students & $1(6.3)$ \\
Premedical students & \\
Study setting & $15(93.8)$ \\
North America & $1(6.3)$ \\
Malaysia & \\
Study design & $15(93.8)$ \\
Cross-sectional & $1(6.3)$ \\
Longitudinal & \\
Measure of burnout & $1(6.3)$ \\
CBI & $11(68.8)$ \\
MBI & $1(6.3)$ \\
ProQOL/CSFT & $3(18.8)$ \\
Single item & \\
\hline
\end{tabular}

$C B I$, Copenhagen Burnout Inventory; $M B I$, Maslach Burnout Inventory; ProQOL/CSFT, Professional Quality of Life/Compassion Satisfaction/Fatigue Self-Test significant differences in burnout among faculty by race/ethnicity when collapsed across non-white categories (Black, Asian, Latinx, South Asian, Middle Eastern, and American Indian); however, these findings were not further disaggregated by type of school or departmental affiliation to disentangle specific effects among faculty within medical fields [42].

\section{Physicians}

Several studies $(n=5)$ assessed burnout among physician populations. An analysis among primary care physicians found that Latinx physicians reported significantly less emotional exhaustion compared to white and non-Latinx nonwhite physicians [43]. Separate analyses among neonatologists found Hispanic physicians reported greater compassion satisfaction, though no statistically significant racial/ethnic differences in compassion fatigue or burnout [44]. Other analyses found no statistically significant differences in burnout by race/ethnicity. Work by Kroll and collaborators found there was no evidence of significant differences in emotional exhaustion, depersonalization, or personal accomplishment by race among a sample of pain physicians [45]. In an assessment among gastroenterologists, Keswani and colleagues found no statistically significant differences in burnout by race/ethnicity [46]. Similarly, no differences were observed in burnout among a sample of physicians in Mississippi [47]. 


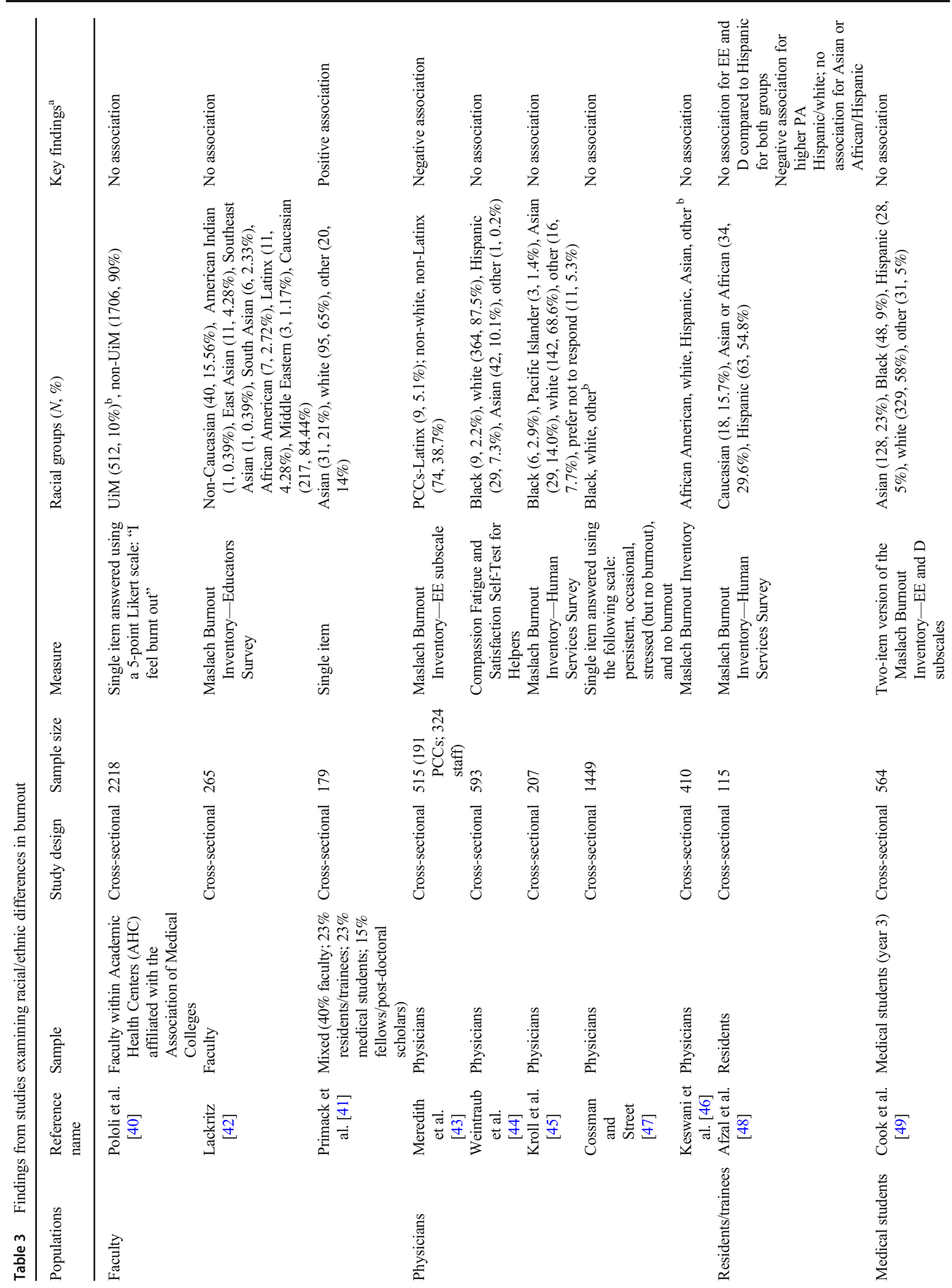




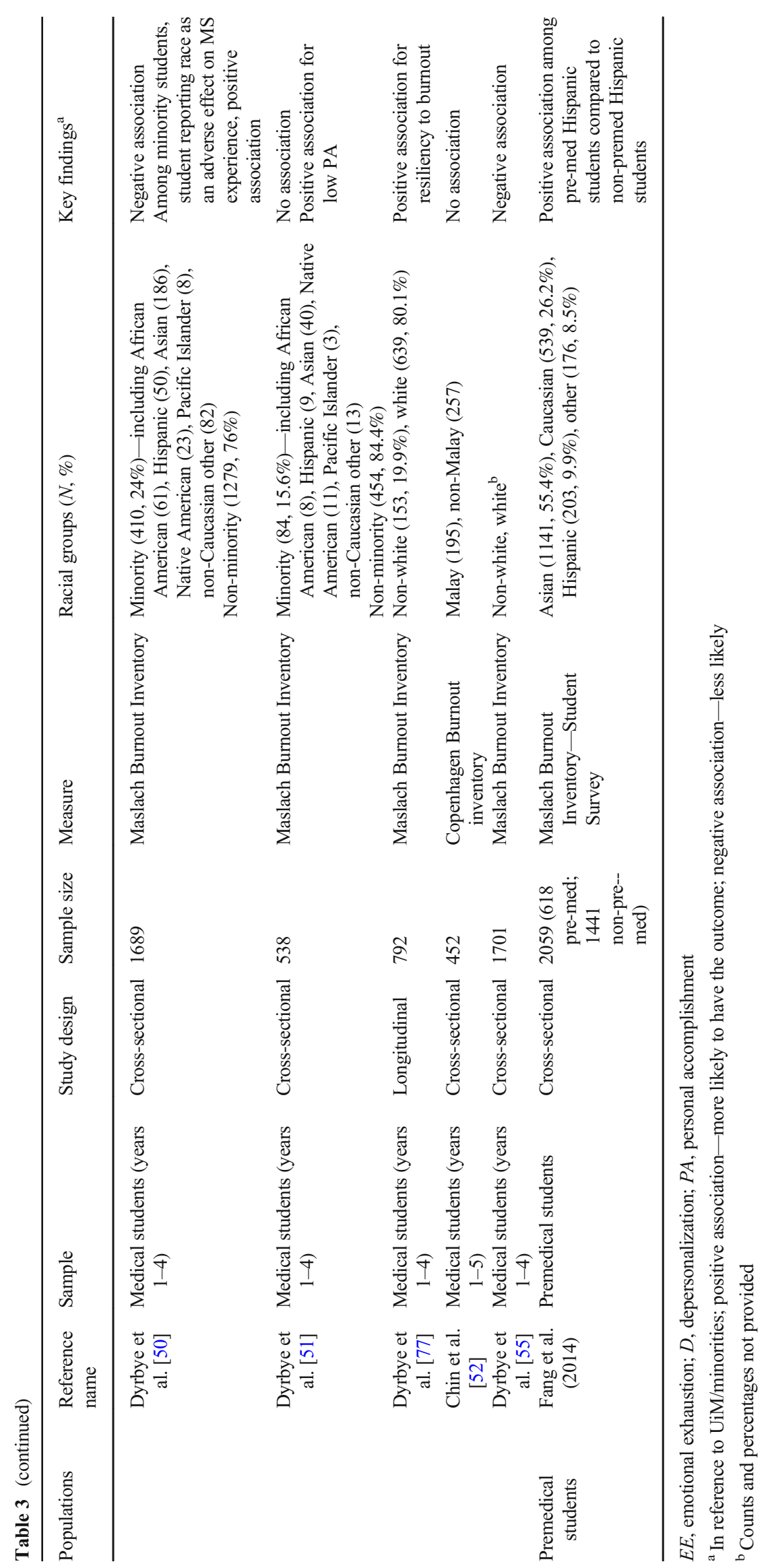




\section{Residents/Trainees}

One study examined racial/ethnic differences in burnout among residents in one academic health center [48]. Differences in burnout were assessed from white, Asian or African, and Hispanic residents from several specialties (i.e., emergency medicine, general surgery, family practice, internal medicine, pediatrics, and obstetrics/gynecology). Findings suggested that there were no statistically significant differences in emotional exhaustion and depersonalization for white or Asian and African residents compared to Hispanic residents; however, the authors observed a significant increase in lower personal accomplishment scores among white residents compared to Hispanic residents in fully adjusted models.

\section{Medical Students}

Studies examining racial/ethnic differences in burnout were primarily conducted among medical students. Of the six studies conducted within populations of medical students, 4 found no evidence of statistically significant differences in the prevalence of burnout by race/ethnicity [49-52]; however, one found that non-minority students were significantly more likely to be burned out, and have higher depersonalization and emotional exhaustion scores [50]. In that study, the authors also found that among minority medical students, experiencing discrimination, prejudice, and isolation was frequently reported and was associated with being more likely to meet the established cutoffs for burnout and having high emotional exhaustion and depersonalization scores [50]. Others found more nuanced associations by race/ethnicity [53-55]. Dyrbye and collaborators found that non-white medical students had greater odds of recovering from burnout and greater odds of resiliency, compared to white medical students [54]. In a separate analysis of medical students from 5 US medical institutions, Dyrbye et al. found nonminority students to have significantly greater odds of burnout compared to minority students [55]. Fang et al. also examined burnout among pre-medical students to understand whether burnout in medical school may be preceded by burnout in pre-medical training [53]. The authors found that Hispanic, pre-medicine students scored higher on cynicism and overall burnout compared to non-pre-medicine Hispanic students; however, no differences were observed for emotional exhaustion or personal accomplishment.

\section{Study Quality and Risk of Bias}

Using the criteria from the quality assessment tool available through the NHLBI, we identified concerns for potential bias among included studies (Fig. 2). While included studies did not provide power calculations, all but two reported either sample size justifications or variance and effect size estimates. The majority of studies were cross-sectional and as such their data collected and subsequent analysis represented a single point in time. Variations in the ability to control for confounding variables were observed - particularly among studies that examined bivariate associations $(n=8)$ compared to those that conducted forms of multivariable regression $(n=8)$, though several important variables were considered in most papers. The majority of studies had sufficient participation rates $(\geq 50 \%)$ and also provided clearly defined and validated outcome measures for all study participants. Based upon these criteria, 15 of 16 studies were classified as having good quality, with one receiving the assessment of fair.

\section{Discussion}

Our review documents the variation in burnout among UiM in existing literature, a contribution to the present understanding of burnout. Included studies were systematically drawn from several databases, used varied instruments to operationalize burnout, and included analyses conducted among several populations (e.g., faculty, medical students).

We observed that studies which assessed the prevalence of burnout among UiM produced inconsistent findings across various populations. For instance, some studies found that UiM experience burnout at lower rates compared to non-UiM [43, 55], while others have found no difference [ $40,42,44-47,49$, $52]$ or greater burnout rate among UiM $[41,53]$. Differences in results may be attributed to the variation of populations assessed — particularly given that many studies were composed of large populations of non-UiM participants - or covariates considered in the regression (e.g., socioeconomic status, specialty, years of practice). Another potential cause of this inconsistency may lie with how burnout is currently measuredparticularly in how it is operationalized, and its accuracy in identifying burnout among UiM. Although most studies utilized the MBI, variations in the number of items or subscales used may lead to differences in results. To our knowledge, the psychometric properties of the MBI have not been examined among UiM populations. However, efforts to understand and measure the experiences of UiM in academic medicine may have the potential to help identify the presentation of burnout and address the sequelae of burnout in these populations.

The review of quantitative literature has highlighted the complexities of examining burnout among UiM. The majority of studies (15 of 16) identified were cross-sectional in design, which are unable to establish temporal relationships between the exposure and outcome (i.e., burnout). Statistical methods of some articles were limited to correlational assessments, $t$ tests, or minimally adjusted linear or logistic regression models which fail to account for additional covariates; however, half of the studies conducted multivariable regressions that adjusted for potential confounders. Some models controlled for factors such as specialty, debt, socioeconomic 
Fig. 2 Quality assessment graph for all 16 studies included using items from the National Heart, Lung, and Blood Institute's (NHLBI) Quality Assessment Tool for Observational Cohort and Cross-Sectional Studies

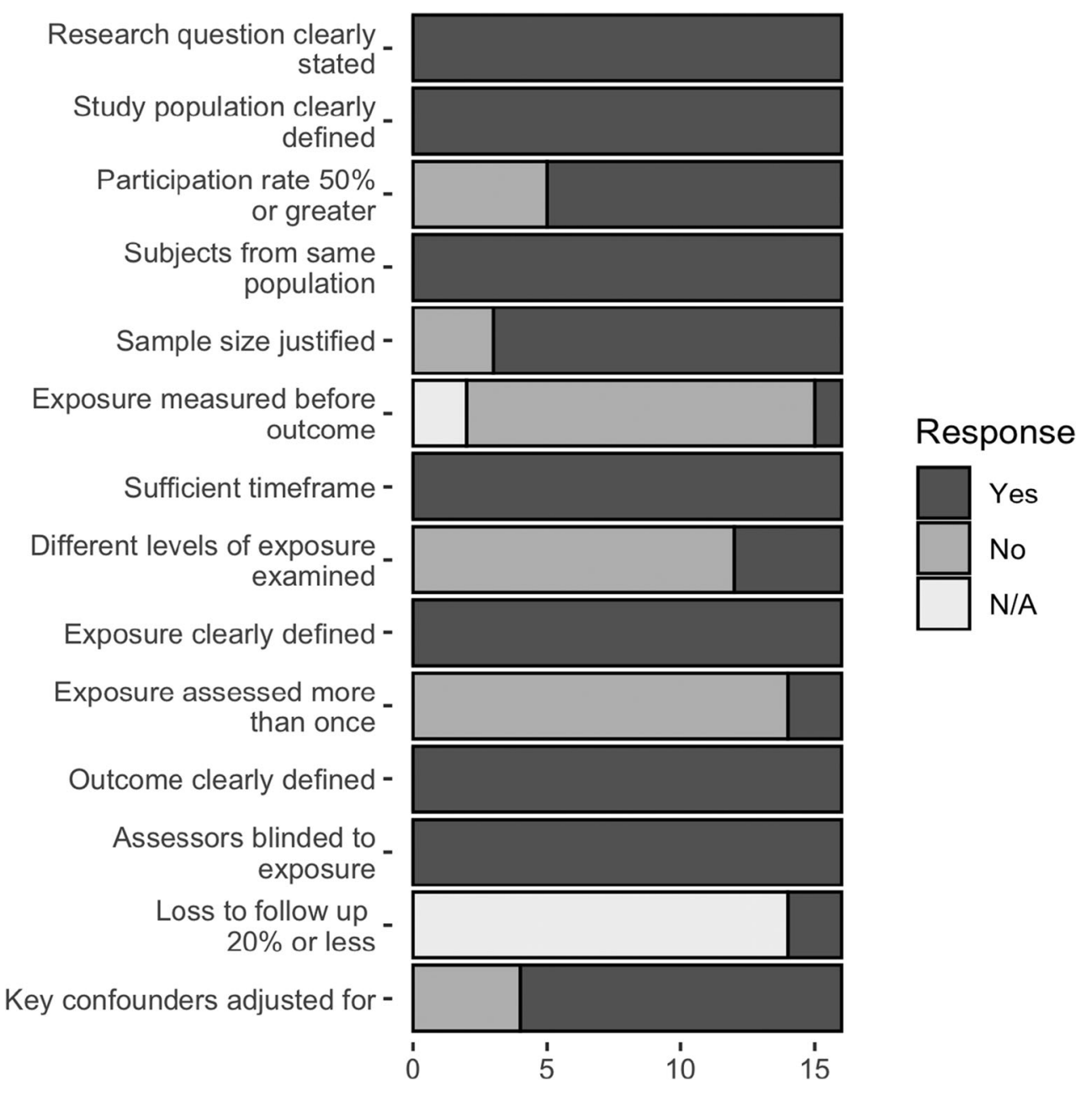

status, and hours worked per week; however, these variables may be correlated with UiM status and mask differences in burnout. Future research on burnout among these populations would benefit from longitudinal designs, to help identify key precursors to burnout symptoms, as well as better consider for possible confounders such as institutional factors or mediators, such as promotion, that may shape trainee or faculty experiences. Longitudinal analysis, beyond the crosssectional approaches, might also lend further insight into what individual and institutional factors facilitate successful transitions from medical school matriculation into practice and/or faculty positions and the role of burnout in that process. Another limitation of the existing literature includes the lack of focus on intersectionality (e.g., LGBTQ+ status, class, gender). For example, examining how marginalized identities intersect and affect burnout across UiM status and gender may provide an understanding of factors shaping burnout development by considering the racialized gender discrimination that UiM women face [24, 56].

Moreover, the conceptualization of burnout among UiM is limited to the scope of existing measures - failing to contextualize the unique experiences and factors pertaining to burnout among minority faculty, students, and physicians, and potentially resulting in underreporting. A majority of studies examining burnout among UiM utilized the MBI to elucidate differences in burnout; however, the utility of the MBI as the gold standard has been questioned by researchers [29, 30]. Studies have highlighted the differential manifestation of burnout across groups and time, finding differences in the utility of the MBI, OLBI, and CBI depending upon which population the measure is utilized in $[27,30,31,33,34]$. Additionally, existing measures of burnout fail to account for critically relevant constructs that structure the lived experiences of UiM, specifically (but not limited to) racism, sexism and gender discrimination, tokenism, isolation or lack of inclusion, resilience, and social support [57-60]. Experiencing discrimination, microaggressions and implicit bias from students, colleagues, or the institution can result in isolation and feelings of not belonging [61, 62]. Experiences of discrimination within academia can manifest as the devaluation of research interests and skill sets of underrepresented minority faculty [61]. Gendered and race-based assumptions of competency, a type of microaggression in the workplace, are documented [63-66]. Specifically, "double doubt" was mentioned 
in reference to Black women faculty, where their competence was questioned as a result of being Black and a woman [63]. URM faculty also face the expectation to take on additional service responsibilities related to diversity, mentoring and advising for URM students - a practice referred to as the "minority tax" [61]. Interviews among Black faculty highlighted differences in the expectations regarding service responsibilities [63]. These additional institutional service responsibilities affect promotion, tenure, and time available to do research (an item heavily weighted in promotion and tenure consideration) [61, 63]. Additionally, while resilience, coping, and social support are useful for combating adversity and have been suggested as reasons for the lower prevalence of burnout among UiM [54], drawing on individual resources yet failing to provide institutional change does little to mitigate and improve burnout outcomes [67]. These experiences may be important to consider when evaluating burnout among UiM at each career stage.

This review was limited by the availability of literature on burnout among UiM $(n=16)$, highlighting the need for more research that examines differences in burnout and its implications by race/ethnicity. Additionally, given that this review restricts inclusion to studies that were published in English, contributions of the literature in other languages were not examined.

To better understand and improve the measurement of burnout among UiM populations, we have identified several points for consideration. Future research should further examine burnout by race/ethnicity, including issues of intersectionality, and contextualize the findings within the structural and systemic barriers specific to UiM populations. The historical and present lived experiences of the racial/ethnic groups that comprise UiM categorizations vary greatly; examining differences in burnout through the lens of UiM as a monolith may mask heterogeneous differences and increase the risk of specific groups being underserved or overlooked. Additionally, in order to not only focus on burnout prevalence, identification, and mitigation-but to also encourage positive outcomes - investigators may benefit from considerations of burnout as existing on a spectrum spanning from thriving (or other forms of well-being) to burnout. Improved measurement of burnout among UiM would benefit from mixed-method approaches where focus groups and/or interviews contribute to the development of instruments to measure burnout-ensuring that experiences and feelings of burnout (and well-being) specific to UiM are included. These measures should also be validated among UiM populations.

Research should also examine multilevel systems (e.g., institution, department, and individual) and the development of interventions that consider the context (e.g., discipline, academic setting, clinical setting), cultural relevance to and heterogeneity of UiM populations in supporting positive psychological outcomes and mitigating burnout.
A growing body of research suggests that what has been described as burnout exists as a consequence of the institutional environment of the healthcare system, and that moral injury - first attributed to healthcare by physicians Talbott and Dean (2018) [68] — better describes the experience of mental distress than burnout. Originally observed among Vietnam War veterans, moral injury is distress caused by engagement in duties (often involuntarily) which were misaligned with personal moral values $[69,70]$. For physicians, competing needs of the healthcare system, such as business imperatives, regulatory and reporting requirements, or policies and practices that may lead to disparate outcomes for certain populations of patients and communities, may lead to moral injury. As research continues to emerge on how best to describe, name, and address physician distress [71-73], it is imperative that the potentially differential experiences among UiM are examined. This will likely be an ongoing topic due to the stress and sacrifices associated with the COVID-19 pandemic, particularly as people of color remain most at risk for exposure and death due to structural factors and racism [74].

There is also a need for research to consider the stability of measurements of burnout. Researchers should assess critical periods of heightened susceptibility to burnout, investigate whether burnout is a transient state or a lasting one, and explore the implications of burnout on faculty, student, and trainee thriving, career and learning outcomes, recruitment, retention, and ways to encourage positive outcomes.

This work underscores the persistent exclusion of UiM individuals from multiple dimensions of study-theoretical and conceptual framing, measurement, analysis, and forging an evidence-based action agenda. This has led to insufficient understanding of the burnout phenomena for UiM students, trainees, physicians, and faculty. It is incumbent that those who both generate and utilize knowledge intentionally address and more fully apply the insights gained through this review in moving toward a more inclusive response to burnout among all members of the academic community.

\section{Conclusion}

Literature has documented that academic faculty, physicians, and medical students experiencing high levels of occupational stress and burnout were more likely to report an intention to leave their current position (or medical school) or reduce hours to part-time work [2, 8, 75-77]. Last, but most importantly, the present body of work speaks to the need for increased focus on burnout of UiM. By omitting the experiences of UiM, we may miss opportunities for efforts that increase the retention, recruitment, well-being, and personal and professional trajectories for UiM physicians, faculty, students, and residents. 
Funding This study was supported by the Aetna Foundation, an independent, charitable and philanthropic affiliate of CVS Health that supports projects to promote wellness, health, and access to high-quality healthcare for everyone. The views presented here are those of the authors and not necessarily those of the Aetna Foundation, its directors, officers, or staff. The authors are indebted to the Foundation for their generosity.

\section{Compliance with Ethical Standards}

Conflict of Interest The authors declare that they have no conflict of interest.

\section{References}

1. Glasheen JJ, Misky GJ, Reid MB, Harrison RA, Sharpe B, Auerbach A. Career satisfaction and burnout in academic hospital medicine. Arch Intern Med. 2011;171(8):782-90. https://doi.org/ 10.1001/archinternmed.2011.153.

2. Shanafelt TD, West CP, Sloan JA, Novotny PJ, Poland GA, Menaker R, et al. Career fit and burnout among academic faculty. Arch Intern Med. 2009;169(10):990-5.

3. Rassolian M, Peterson LE, Fang B, Knight HC, Peabody MR, Baxley EG, et al. Workplace factors associated with burnout of family physicians. JAMA Intern Med. 2017;177(7):1036-8.

4. Shanafelt TD, Boone S, Tan L, Dyrbye LN, Sotile W, Satele D, et al. Burnout and satisfaction with work-life balance among US physicians relative to the general US population. JAMA Intern Med. 2012;172(18):1377-85. https://doi.org/10.1001/ archinternmed.2012.3199.

5. Shanafelt TD, Sloan JA, Habermann TM. The well-being of physicians. Am J Med. 2003;114(6):513.

6. Rothenberger DA. Physician burnout and well-being: a systematic review and framework for action. Dis Colon Rectum. 2017;60(6): 567-76.

7. Schindler BA, Novack DH, Cohen DG, Yager J, Wang D, Shaheen $\mathrm{NJ}$, et al. The impact of the changing health care environment on the health and well-being of faculty at four medical schools. Acad Med. 2006;81(1):27-34.

8. Lowenstein SR, Fernandez G, Crane LA. Medical school faculty discontent: prevalence and predictors of intent to leave academic careers. BMC Med Educ. 2007;7(1):37.

9. Windover AK, Martinez K, Mercer MB, Neuendorf K, Boissy A, Rothberg MB. Correlates and outcomes of physician burnout within a large academic medical center. JAMA Intern Med. 2018;178(6):856-8.

10. Shanafelt TD, Hasan O, Dyrbye LN, Sinsky C, Satele D, Sloan J et al., editors. Changes in burnout and satisfaction with work-life balance in physicians and the general US working population between 2011 and 2014. Mayo Clinic Proceedings; 2015: Elsevier.

11. Maslach C, Jackson SE. The measurement of experienced burnout. J Organ Behav. 1981;2(2):99-113.

12. Rotenstein LS, Torre M, Ramos MA, Rosales RC, Guille C, Sen S, et al. Prevalence of burnout among physicians: a systematic review. Jama. 2018;320(11):1131-50.

13. Ishak WW, Lederer S, Mandili C, Nikravesh R, Seligman L, Vasa $\mathrm{M}$, et al. Burnout during residency training: a literature review. J Grad Med Educ. 2009;1(2):236-42. https://doi.org/10.4300/ JGME-D-09-00054.1.

14. Dandar V, Grigsby RK, Bunton S. Burnout among US medical school faculty. Anal Brief. 2019;19(1):1.

15. Dewa CS, Loong D, Bonato S, Thanh NX, Jacobs P. How does burnout affect physician productivity? A systematic literature review. BMC Health Serv Res. 2014;14(1):325.
16. Dyrbye LN, Shanafelt TD. Physician burnout: a potential threat to successful health care reform. Jama. 2011;305(19):2009-10.

17. Oreskovich MR, Kaups KL, Balch CM, Hanks JB, Satele D, Sloan $\mathrm{J}$, et al. Prevalence of alcohol use disorders among American surgeons. Arch Surg. 2012;147(2):168-74.

18. Shanafelt TD, Balch CM, Dyrbye L, Bechamps G, Russell T, Satele D, et al. Special report: suicidal ideation among American surgeons. Arch Surg. 2011;146(1):54-62.

19. Odom KL, Roberts LM, Johnson RL, Cooper LA. Exploring obstacles to and opportunities for professional success among ethnic minority medical students. Acad Med. 2007;82(2):146-53.

20. Hardeman RR, Przedworski JM, Burke S, Burgess DJ, Perry S, Phelan S, et al. Association between perceived medical school diversity climate and change in depressive symptoms among medical students: a report from the medical student CHANGE study. J Natl Med Assoc. 2016;108(4):225-35.

21. Peterson NB, Friedman RH, Ash AS, Franco S, Carr PL. Faculty self-reported experience with racial and ethnic discrimination in academic medicine. J Gen Intern Med. 2004;19(3):259-65.

22. Nunez-Smith M, Pilgrim N, Wynia M, Desai MM, Jones BA, Bright C, et al. Race/ethnicity and workplace discrimination: results of a national survey of physicians. J Gen Intern Med. 2009;24(11): 1198-204.

23. Association of American Medical Colleges. Underrepresented in Medicine Definition. 2004. https://www.aamc.org/initiatives/urm/. Accessed 22 July 2019.

24. National Academies of Sciences, Engineering, and Medicine. Promising practices for addressing the underrepresentation of women in science, engineering, and medicine: Opening Doors. Washington: The National Academies Press; 2020.

25. Freudenberger HJ. The staff burn-out syndrome in alternative institutions. Psychotherapy: Theory, Research \& Practice. 1975;12(1): 73.

26. Maslach C, Leiter MP. Understanding the burnout experience: recent research and its implications for psychiatry. World Psychiatry. 2016;15(2):103-11. https://doi.org/10.1002/wps.20311.

27. Green DE, Walkey FH, Taylor AJ. The three-factor structure of the Maslach Burnout Inventory: a multicultural, multinational confirmatory study. J Soc Behav Pers. 1991;6(3):453.

28. Rafferty JP, Lemkau JP, Purdy RR, Rudisill JR. Validity of the Maslach Burnout Inventory for family practice physicians. J Clin Psychol. 1986;42(3):488-92.

29. Demerouti E, Bakker AB, Nachreiner F, Schaufeli WB. The job demands-resources model of burnout. J Appl Psychol. 2001;86(3): 499-512.

30. Kristensen TS, Borritz M, Villadsen E, Christensen KB. The Copenhagen Burnout Inventory: a new tool for the assessment of burnout. Work Stress. 2005;19(3):192-207.

31. Demerouti E, Bakker AB, Vardakou I, Kantas A. The convergent validity of two burnout instruments: a multitrait-multimethod analysis. Eur J Psychol Assess. 2003;19(1):12-23.

32. Koeske GF, Koeske RD. Construct validity of the Maslach Burnout Inventory: a critical review and reconceptualization. J Appl Behav Sci. 1989;25(2):131-44. https://doi.org/10.1177/ 0021886389252004 .

33. Halbesleben JR, Demerouti E. The construct validity of an alternative measure of burnout: investigating the English translation of the Oldenburg Burnout Inventory. Work Stress. 2005;19(3):208-20.

34. Reis D, Xanthopoulou D, Tsaousis I. Measuring job and academic burnout with the Oldenburg Burnout Inventory (OLBI): factorial invariance across samples and countries. Burn Res. 2015;2(1):818. https://doi.org/10.1016/j.burn.2014.11.001.

35. Schaufeli WB, van Dierendonck D. De Utrechtse Burnout Schaal, handleiding [Utrecht Burnout Scale: Test manual]. Lisse: The Netherlands Swets Test Services; 2000. 
36. Stamm BH. Measuring compassion satisfaction as well as fatigue: developmental history of the Compassion Satisfaction and Fatigue Test. 2002.

37. Pines AM, Aronson E. Career burnout. New York: Free Press; 1988.

38. Veritas Health Innovation. Covidence systematic review software. VIC: Veritas Health Innovation Melbourne; 2017.

39. National Heart Lung and Blood Institute. Quality assessment tool for observational cohort and cross-sectional studies. Bethesda: National Institutes of Health, Department of Health and Human Services; 2014. p. 103-11.

40. Pololi LH, Evans AT, Civian JT, Gibbs BK, Coplit LD, Gillum LH, et al. Faculty vitality-surviving the challenges facing academic health centers: a national survey of medical faculty. Acad Med. 2015;90(7):930-6.

41. Primack BA, Dilmore TC, Switzer GE, Bryce CL, Seltzer DL, Li J, et al. Burnout among early career clinical investigators. Clin Transl Sci. 2010;3(4):186-8. https://doi.org/10.1111/j.1752-8062.2010. 00202.x.

42. Lackritz JR. Exploring burnout among university faculty: incidence, performance, and demographic issues. Teach Teach Educ. 2004;20(7):713-29.

43. Meredith LS, Hackbarth NS, Darling J, Rodriguez HP, Stockdale $\mathrm{SE}$, Cordasco KM, et al. Emotional exhaustion in primary care during early implementation of the VA's medical home transformation: Patient-aligned Care Team (PACT). Med Care. 2015;53(3): 253-60. https://doi.org/10.1097/mlr.0000000000000303.

44. Weintraub AS, Geithner EM, Stroustrup A, Waldman ED. Compassion fatigue, burnout and compassion satisfaction in neonatologists in the US. J Perinatol: official journal of the California Perinatal Association. 2016;36(11):1021-6. https://doi.org/10. 1038/jp.2016.121.

45. Kroll H, Macaulay T, Jesse M. A Preliminary survey examining predictors of burnout in pain medicine physicians in the United States. Pain Physician. 2016;19(5):E689.

46. Keswani RN, Taft TH, Coté GA, Keefer L. Increased levels of stress and burnout are related to decreased physician experience and to interventional gastroenterology career choice: findings from a US survey of endoscopists. Am J Gastroenterol. 2011;106(10): $1734-40$.

47. Cossman JS, Street D. Mississippi burnout part II: satisfaction, autonomy and work/family balance. J Miss State Med Assoc. 2009;50(10):338-45.

48. Afzal KI, Khan FM, Mulla Z, Akins R, Ledger E, Giordano FL. Primary language and cultural background as factors in resident burnout in medical specialties: a study in a bilingual US city. South Med J. 2010;103(7):607-15.

49. Cook AF, Arora VM, Rasinski KA, Curlin FA, Yoon JD. The prevalence of medical student mistreatment and its association with burnout. Acad Med: journal of the Association of American Medical Colleges. 2014;89(5):749-54.

50. Dyrbye LN, Thomas MR, Eacker A, Harper W, Massie FS Jr, Power DV, et al. Race, ethnicity, and medical student well-being in the United States. Arch Intern Med. 2007;167(19):2103-9. https://doi.org/10.1001/archinte.167.19.2103.

51. Dyrbye LN, Thomas MR, Huschka MM, Lawson KL, Novotny PJ, Sloan JA, et al. A multicenter study of burnout, depression, and quality of life in minority and nonminority US medical students. Mayo Clin Proc. 2006;81(11):1435-42. https://doi.org/10.4065/81. 11.1435 .

52. Chin RWA, Chua YY, Chu MN, Mahadi NF, Yusoff MSB, Wong MS, et al. Prevalence of burnout among Universiti Sains Malaysia Medical Students. Educ Med J. 2016;8(3):61-74.

53. Fang DZ, Young CB, Golshan S, Moutier C, Zisook S. Burnout in premedical undergraduate students. Acad Psychiatry : the journal of the American Association of Directors of Psychiatric Residency
Training and the Association for Academic Psychiatry. 2012;36(1):11-6. https://doi.org/10.1176/appi.ap.10080125.

54. Dyrbye LN, Power DV, Massie FS, Eacker A, Harper W, Thomas MR, et al. Factors associated with resilience to and recovery from burnout: a prospective, multi-institutional study of US medical students. Med Educ. 2010;44(10):1016-26. https://doi.org/10.1111/j. 1365-2923.2010.03754.x.

55. Dyrbye LN, Thomas MR, Harper W, Massie FS, Power DV, Eacker A, et al. The learning environment and medical student burnout: a multicentre study. Med Educ. 2009;43(3):274-82. https://doi.org/10.1111/j.1365-2923.2008.03282.x.

56. Williams J, Phillips K, Hall E. Double jeopardy? Gender bias against women of color in science. UC Hastings College of the Law; 2014. Published online at www.worklifelaw.org. Accessed 1 Apr 2020.

57. Pololi L, Cooper LA, Carr P. Race, disadvantage and faculty experiences in academic medicine. J Gen Intern Med. 2010;25(12): 1363-9.

58. Carr PL, Palepu A, Szalacha L, Caswell C, Inui T. 'Flying below the radar': a qualitative study of minority experience and management of discrimination in academic medicine. Med Educ. 2007;41(6):601-9.

59. Cora-Bramble D, Zhang K, Castillo-Page L. Minority faculty members' resilience and academic productivity: are they related? Acad Med. 2010;85(9):1492-8.

60. Hassouneh D, Lutz KF, Beckett AK, Junkins EP Jr, Horton LL. The experiences of underrepresented minority faculty in schools of medicine. Medi Educ Online. 2014;19(1):24768.

61. Zambrana RE, Harvey Wingfield A, Lapeyrouse LM, Dávila BA, Hoagland TL, Valdez RB. Blatant, subtle, and insidious: URM faculty perceptions of discriminatory practices in predominantly White institutions. Sociol Inq. 2017;87(2):207-32.

62. Zambrana RE, Valdez RB, Pittman CT, Bartko T, Weber L, ParraMedina D. Workplace stress and discrimination effects on the physical and depressive symptoms of underrepresented minority faculty. Stress Health. 2020. https://doi.org/10.1002/smi.2983.

63. Griffin KA, Bennett JC, Harris J. Analyzing gender differences in black faculty marginalization through a sequential mixed-methods design. New Dir Inst Res. 2011;2011(151):45-61.

64. Turner CSV, González JC. Faculty women of color: the critical nexus of race and gender. J Divers High Educ. 2011;4(4):199-211.

65. Turner CSV, Myers SL Jr, Creswell JW. Exploring underrepresentation: the case of faculty of color in the Midwest. J High Educ. 1999;70(1):27-59.

66. Zambrana RE, Dávila BA, Espino MM, Lapeyrouse LM, Valdez $\mathrm{RB}$, Segura DA. Mexican American faculty in research universities: can the next generation beat the odds? Sociol Race Ethn. 2017;3(4): 458-73.

67. Leiter M. Preventing workplace burnout: why resilience is not enough. InPsych: The Bulletin of the Australian Psychological Society. 2018;40(3):24.

68. Talbot SG, Dean W. Physicians aren't 'burning out.' They're suffering from moral injury. Stat. 2018;7(26):18.

69. Litz BT, Stein N, Delaney E, Lebowitz L, Nash WP, Silva C, et al. Moral injury and moral repair in war veterans: a preliminary model and intervention strategy. Clin Psychol Rev. 2009;29(8):695-706.

70. Friedman MJ. Post-Vietnam syndrome: recognition and management. Psychosomatics. 1981;22(11):931-42.

71. Dean W, Simon Talbot AD. Reframing clinician distress: moral injury not burnout. Fed Pract. 2019;36(9):400-2.

72. Mehta P, Mehta J. Reframing clinician distress: moral injury not burnout. Fed Pract. 2019;36(11):504-6.

73. Padgett L, Ascensao JL. Reframing clinician distress: moral injury not burnout. Fed Pract: for the Health Care Professionals of the VA, Dod, and PHS. 2019;36(11):502-4. 
74. Cowger TL, Davis BA, Etkins OS, Makofane K, Lawrence JA, Bassett MT, et al. Comparison of weighted and unweighted population data to assess inequities in coronavirus disease 2019 deaths by race/ethnicity reported by the US Centers for Disease Control and Prevention. JAMA Netw Open. 2020;3(7):e2016933.

75. Goitein L, Shanafelt TD, Nathens AB, Curtis JR. Effects of resident work hour limitations on faculty professional lives. J Gen Intern Med. 2008;23(7):1077-83.

76. Golub JS, Johns MM III, Weiss PS, Ramesh AK, Ossoff RH. Burnout in academic faculty of otolaryngology - head and neck surgery. Laryngoscope. 2008;118(11):1951-6.
77. Dyrbye LN, Thomas MR, Power DV, Durning S, Moutier C, Massie FS, et al. Burnout and serious thoughts of dropping out of medical school: a multi-institutional study. Acad Med. 2010;85(1): 94-102. https://doi.org/10.1097/acm.0b013e3181c46aad.

Publisher's Note Springer Nature remains neutral with regard to jurisdictional claims in published maps and institutional affiliations. 